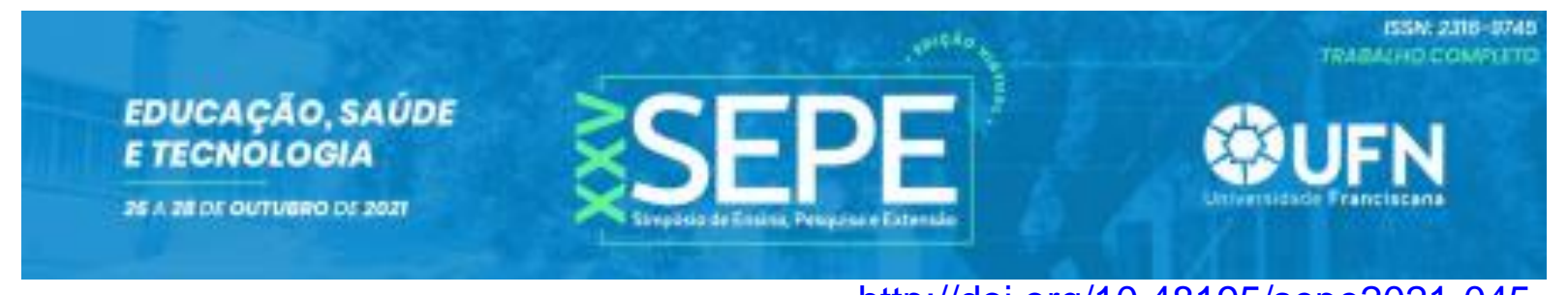

\title{
O TRABALHO DO PSICÓlOGO NA ÊNFASE DE PROMOÇÃO E PREVENÇÃO EM SAÚDE
}

\section{Laura Vieira Moura'; Márcia Elisa Jager²}

\section{RESUMO}

Objetiva-se descrever a experiência acadêmica prática de estágio na ênfase de prevenção e promoção da saúde realizada no ambulatório de especialidades do Hospital Casa de Saúde. Este estágio propõe o exercício da prática profissional alicerçada num conceito ampliado de saúde, que compreende uma visão integral e contextualizada do ser humano em suas relações sociais, econômicas, políticas, ambientais e biológicas. Foram realizados atendimentos individuais, práticas de acolhimento, criação de cartazes informativos e fluxograma da rede de atendimentos psicológicos. Conclui-se que os conceitos vistos teoricamente podem ser vividos de forma prática nos estágios e que o atendimento psicológico no ambulatório prevê que o paciente se torne ativo na promoção da própria saúde.

Palavras-chave: Ambulatório; Atendimento psicológico; Estágio.

Eixo Temático: Atenção Integral e Promoção à Saúde (AIPS)

\section{INTRODUÇÃO}

O presente relatório parcial de estágio se refere à prática de estágio específico com ênfase em psicologia e processos de prevenção e promoção da saúde vinculado ao curso de Psicologia da Universidade Franciscana. Este estágio proporcionou a experiência da prática de intervenção em Psicologia em um ambulatório anexo ao Hospital Casa de Saúde, vinculado ao Sistema Único de Saúde. Atualmente neste ambulatório são realizados acompanhamentos psicológicos, atendimentos interdisciplinares em saúde mental, orientação em saúde mental para a equipe de saúde, apoio ao setor da psicologia do Hospital Casa De Saúde em urgências

\footnotetext{
1 Autor/Apresentador - Universidade Franciscana- UFN. laura.moura@ufn.edu.br

${ }^{2}$ Professora Orientadora - Universidade Franciscana- UFN. marcia.jager@ufn.edu.br
} 
clínicas e avaliação psicológica. Foram realizados atendimentos psicológicos breves

com foco na avaliação e compreensão da demanda do usuário. Também foi construída uma lista de encaminhamentos psicológicos com valor social na cidade de Santa Maria. A lista inclui os serviços disponíveis nas universidades do município. A prática do psicólogo em contextos de promoção e prevenção de saúde torna evidente o compromisso destes profissionais em aceitar os indivíduos na sua subjetividade. Seu papel é mais do que receber, triar ou avaliar; é ir além. É proporcionar uma escuta qualificada, ser suporte, rever a história do outro, acolher, cuidar, estar atento aos sinais, buscar de forma humanizada o entendimento e solução de problemas, respeitando os limites profissionais e institucionais. O psicólogo também pode orientar, esclarecer sobre doenças, prevenir, educar, conversar, como um processo generalizado de prevenção cuja intenção é informar.

Bleger (1992) ainda alerta que "a função do psicólogo não deve ser basicamente a terapia e sim a saúde pública"(p.20), sendo assim, o psicólogo precisa ocupar um lugar junto a toda equipe de saúde, pois neste contexto existem muitas necessidades, mas também muitas possibilidades de se beneficiar um maior número de pessoas, enquanto na clínica individualizada este trabalho é mais lento e atende a uma minoria. A promoção em saúde se refere a medidas que não dirigem a uma determinada doença ou desordem, mas servem para aumentar a saúde e bem-estar no geral, buscando melhoria na qualidade de vida. As ações de promoção de saúde são iniciativas que permitem de alguma forma melhorar a condição de saúde dos indivíduos ou das populações. Podem ser ações coletivas ou individuais. Enquanto a prevenção de saúde exige uma ação antecipada, baseado no conhecimento da história natural a fim de tornar improvável o progresso posterior da doença, um exemplo de prevenção seria a vacinação da população contra a covid-19 (CZERESNIA, 2009).

O objetivo deste relatório é descrever a experiência acadêmica de uma prática de estágio em prevenção e promoção da saúde realizada no ambulatório do Hospital Casa de Saúde. Esta prática está vinculada à prática supervisionada do Estágio Específico I do curso de Psicologia da Universidade Franciscana e ocorreu de forma 
individual. Este estágio tem como objetivo geral propor o exercício da prática

profissional alicerçada num conceito ampliado de saúde, que compreende uma visão integral e contextualizada do ser humano em suas relações sociais, econômicas, políticas, ambientais e biológicas. Essa ênfase pressupõe, ainda, a articulação entre saberes da psicologia com outras áreas do conhecimento, de maneira a desenvolver competências técnicas, éticas e de humanização na atenção à saúde, voltadas para assistência, prevenção e promoção de saúde. Inclui temáticas como: saúde mental, atenção primária à saúde, sistema jurídico, modelos de atenção e organização dos serviços de saúde, epidemiologia e princípios do Sistema Único de Saúde (SUS).

\section{METODOLOGIA}

Este trabalho fundamenta-se no relato de experiências vivências no estágio supervisionado, o qual ocorreu no ambulatório de especialidades anexo ao Hospital Casa de Saúde. O período mínimo de duração deste estágio é de um ano letivo. Sua carga horária total semestral é de 220 horas, distribuídas em 08 horas/aula semanais de prática e 02 horas/aula semanais de supervisão. A carga horária semestral de prática equivale a 180 horas/aula nas quais são desenvolvidas a prática no local, elaboração de plano de estágio, elaboração de diários de bordo semanais, o estudo de textos indicados pela professora, a participação em eventos / lives / grupos de estudos, participação em supervisão coletiva e elaboração de relatório final de estágio. A carga horária semestral de supervisões acadêmicas equivale a 40 horas/aula.

O estágio ocorreu do período de março até julho de 2021 totalizando 219 horas de prática. As supervisões acadêmicas ocorreram semanalmente, de forma síncrona, via Teams. Como forma de aproveitar o espaço de supervisão como um momento coletivo de aprendizagem, elas foram realizadas em grupo. Para cada supervisão foram elaborados diários de bordo compartilhados com a professora supervisora, via google drive.

\section{RESULTADOS E DISCUSSÕES}

Ambulatório é um local onde se presta assistência a pacientes, sem regime de 
internação. Desta forma, os atendimentos psicológicos são realizados de forma breve, com foco na avaliação e compreensão da demanda. Se a demanda for de psicoterapia o usuário deve ser encaminhado a algum serviço da rede, já que o serviço de psicologia oferecido no ambulatório não comporta psicoterapia. (BRASIL, 1983).

O ambulatório em que fora realizado o presente estágio fica anexo ao Hospital Casa De Saúde, na cidade de Santa Maria no Rio Grande Do Sul. O ambulatório funciona há poucos anos e por não ser exclusivo de saúde mental contempla diversas especialidades para atendimento, sendo eles: equipe CLASM de anestesiologia; 3 odontólogos bucomaxilofacial; 2 médicos cirurgiões gerais; 1 médico pediatra; 1 médico cirurgião plástico; 1 médico da clínica da dor; 4 médicos ginecologistas; 2 fonoaudiólogos, 1 nutricionista; 3 médicos otorrinolaringologistas; 1 médico proctologista; 1 psicólogo; 4 médicos psiquiatras; 2 médicos responsáveis pelo puerpério; 2 médicos responsáveis pelo pré-natal; 3 médicos urologistas; 3 médicos vasculares; 4 médicos cardiologistas; 2 médicos dermatologistas; 2 médicos endocrinologistas; 3 médicos gastroenterologistas; 1 médico hematologista; 2 médicos nefrologistas; 1 médico neurologista; 2 médicos ortopedistas; 3 médicos pneumologistas e 2 médicos reumatologistas. Além dos profissionais, o ambulatório conta com estagiários da Universidade Franciscana, onde as práticas de estágios curriculares são orientadas por professores da instituição.

O local recebe pacientes encaminhados pela secretaria da saúde do município. Os atendimentos e/ou encaminhamentos para a psicologia são feitos pelos profissionais que atendem no ambulatório, Hospital Casa de Saúde e UPA 24H. As consultas das especialidades se dividem em: primeira consulta para e retorno para exames e pós-operatório.

Acerca da estrutura física do local, a distribuição ocorre em 3 andares através de salas pequenas e arejadas, recepção ampla, banheiros grandes, um elevador e escadas espaçosas. As salas onde os atendimentos foram realizados estão localizadas no subsolo e são chamadas "salas de estudo", por isso possuem mesa grande, diversas cadeiras, quadro e armários.

O horário de funcionamento do local é das $8 \mathrm{~h}$ às $17 \mathrm{~h}$, sem fechar ao meio-dia 
EDUCAÇĀO, SAŪDE

ETECNOLOGIA

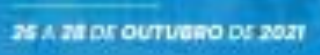

Vale ressaltar que no ambulatório não estão sendo recebidos pacientes com

coronavírus, além de ter uma triagem COVID antes de ingressar no local, a fim de avaliar os sintomas da doença. Sendo assim, para que houvesse segurança nos atendimentos realizados, todos os pacientes descritos neste relatório passaram pela triagem antes de cada encontro.

Visto que, a função do psicólogo na ênfase de promoção e prevenção pode contemplar a orientação e esclarecimento de doenças, durante os primeiros meses do presente estágio fora desenvolvido cartazes que ressaltam a importância de manter o distanciamento social dentro do ambulatório, principal no elevador. Além da criação dos cartazes, foi desenvolvido um material para se ter uma estrutura de redes de atendimentos psicológicos na cidade de Santa Maria. Para a construção do material foram selecionadas as Universidades da cidade que oferecem atendimento ao público, e então feito o contato. As universidades e projetos que prestam assistência ao público são: Projeto Transformar- CEIP UFSM; Clinicog - CEIP UFSM; CEPPSI - ULBRA; PAIP - UFN; Clínica Escola - UFN; e Clínica Escola - FISMA. O material desenvolvido será disponibilizado para estagiários do ambulatório para que possam fazer encaminhamento dos pacientes que precisam de psicoterapia.

Outras práticas que estão presentes na atuação do psicólogo no contexto do SUS são as de acolhimento e humanização, sendo essas questões transversais que apareceram nas práticas deste estágio. O acolhimento é uma ação que deve existir em todas as relações de cuidado, entre trabalhadores de saúde e usuários, na prática de receber e escutar as pessoas, e deve ser estabelecido como uma prática que possibilita a humanização do cuidado, além de ampliar o acesso da população aos serviços de saúde, assegurando a resolução dos problemas. Sendo assim, pode-se dizer que essa prática está presente em todas as relações de cuidado e pode se configurar de diferentes. À vista disso, em vez de indagar se, em algum serviço, existe ou não o uso da ferramenta acolhimento, talvez seja mais pertinente avaliar como ele se concretiza ou é empregado. $O$ acolhimento se refere somente a uma prática isolada, mas sim a uma postura ética dos profissionais de saúde frente ao cuidado e validação das necessidades do usuário. Ele é uma prática que está 
para além de uma demanda emergente. O acolhimento é transmitido menos no discurso sobre ele do que nas práticas propriamente ditas (BRASIL, 2011; LOPES, 2014).

Quando a intervenção contemplou atendimentos clínicos individuais, o presente estágio foi realizado seguindo os princípios da Terapia do Esquema. Essa terapia é considerada inovadora e integradora, já que Jeffrey Young a desenvolveu com a intenção de superar as limitações identificadas na terapia cognitiva tradicional. Desta forma, Young $(1990,1999)$ formulou a hipótese de que alguns desses esquemas podem estar no centro de transtornos de personalidade, problemas caracterológicos mais leves e muitos transtornos do Eixo 1.

Para as demais intervenções realizadas no contexto do ambulatório, visando a prevenção e promoção da saúde, as referências foram buscadas na literatura do campo da saúde coletiva. No contexto da prevenção e promoção da saúde, sabe-se da importância de um trabalho em equipe. Neste, cada profissional deve desenvolver suas atribuições e responsabilidades para com os pacientes, mas nenhum saber pode sobressair-se ao outro e desintegrar o paciente em partes. $O$ trabalho em equipe na saúde ocorre com vários profissionais de diversas formações na área da saúde, cada especialidade compõe o campo de saber que visa o bem-estar biopsicossocial dos sujeitos. O papel da psicologia no trabalho em equipes de saúde é de extrema importância e essa profissão não deve se destinar somente ao atendimento individual, mas sim compor o campo dos saberes interdisciplinares. Os profissionais entendem que é fundamental um trabalho em equipe, sendo fundamental para propiciar um melhor entendimento do quadro do paciente, bem como para a promoção de saúde e bem-estar dele. Um estudo realizado recentemente por Bonaldi et al. (2007), mostra na fala de diversos profissionais questões de hierarquia que ainda são presentes e acabam por atrapalhar a articulação entre os membros da equipe. Diversas vezes a prática de um profissional torna invisível a prática do outro e faz com que apareça na equipe apenas os diferentes especialistas de uma mesma categoria, como por exemplo, da classe médica. Para os profissionais da área da psicologia sabe-se que é preciso adaptar se ao contexto, sendo este diferente da clínica particular e logo a prática

exige 
também uma adequação de abordagem a ser executada, pois o contexto em que se

encontra um paciente em um ambulatório do SUS difere do perfil do paciente de consultório ou clínica de psicologia privada.

Quanto aos atendimentos clínicos, foi feito contato com cerca de nove pessoas, destas, duas se mantem em atendimento até o presente momento, três marcaram atendimento e por terem faltado aos atendimentos por três vezes consecutivas e sem justificativa foram desligadas do serviço e as demais não tinham disponibilidade de horário e foram encaminhadas para outros estagiários.

Dentre os pacientes que estão em atendimento, temos a paciente $\mathrm{F}$ e o paciente JP. O primeiro atendimento com a paciente $F$ ocorreu no dia 12/05/21. Foram realizados três atendimentos com foco em avaliação psicológica. Por conta de seu histórico clínico e psiquiátrico a paciente foi direcionada ao estágio com foco em promoção em prevenção em saúde. Até o dia 07/07/21 foram realizados nove atendimentos. A paciente faltou aproximadamente três vezes, porém justificou todas elas. A paciente é uma mulher, 44 anos, viúva, portadora de fibromialgia e obesidade. A principal demanda da usuária é de emagrecimento. As dores e a impossibilidade de realizar algumas atividades por conta da fibromialgia são outra demanda aparente. A paciente relata arrancar os cabelos até fazer grandes feridas na cabeça, que normalmente infeccionam. Outra questão é a de automedicação, pois a paciente utiliza diversos remédios diariamente sem controle algum. Ela relata que precisa estar sempre em alerta e que sua mente não para. Há a hipótese diagnóstica de tricotilomania e transtorno de ansiedade generalizada. Foi feito encaminhamento para a usuária ao endocrinologista, para que possa ver questões hormonais e depois ser direcionada ao nutricionista. Também foi feito encaminhamento ao cardiologista e ginecologista para serem feitos exames de rotina. Todos os encaminhamentos foram internos. A paciente relata possuir esquecimento, mas quando questionado foi entendido que o que ocorrem são trocas de palavras. No último atendimento (07/07/21) foi construída com a paciente uma lista de informações e problemas que ela poderia dizer aos médicos endocrinologista, cardiologista e ginecologista. Ela relatou várias vezes o quanto achou boa a experiência de organizar em tópicos suas questões, disse 
muita clareza. Enquanto a paciente escrevia, foi notório que também ocorre a troca de letras.

Outro paciente que está em acompanhamento é JP, de 04 anos. O primeiro contato foi feito com a mãe e então marcado o primeiro atendimento, onde os dois deveriam comparecer. O primeiro atendimento ocorreu no dia 23/06/21. Segundo a mãe, suas demandas são de mudança de rotina, dificuldade para dormir e falta de contato com o pai. A mãe, JP e os irmãos se mudaram para a casa da avó materna após um episódio de agressão entre o pai de JP e o irmão mais velho. No dia 30/06/21 foi realizado o segundo atendimento com o paciente. É notório um apego muito grande do usuário com a mãe. Foram realizados apenas dois atendimentos com a criança, sendo um deles com a presença da mãe. Fora marcado um atendimento somente com a mãe, porém ela não compareceu e justificou sua falta.

A prática de estágio baseou-se no Código de Ética Profissional do Psicólogo (CFP, 2005). Durante a pandemia, algumas práticas ocorreram de forma síncrona / remota conforme orienta o documento "Práticas de estágios remotos em Psicologia no contexto da pandemia da Covid-19", elaborado pelo Conselho Federal de Psicologia a partir da portaria MEC no 544, de 2020 (CFP, 2020) e outros documentos importantes. O documento expõe várias possibilidades de práticas em psicologia de acordo com os processos de trabalho.

\section{CONCLUSÃO}

Foram diversos aspectos aprendidos ao longo do semestre. Por conta de estar fazendo no mesmo momento do estágio a disciplina de Saúde Coletiva, alguns conceitos aprendidos puderem ser vistos de formas práticas no estágio. $\mathrm{O}$ acolhimento foi um destes aspectos. Outro aspecto aprendido ao longo da prática de estágio que cabe salientar seria o aprendizado de atendimento psicológico em ambulatório. O atendimento deve ser breve com foco na avaliação e compreensão de demanda do usuário, além de que por conta de o enfoque do estágio ser em promoção e prevenção em saúde é necessário a criação de um vínculo institucional com o paciente, a fim de desenvolver seu senso de autonomia e torná-lo um agente 
ativo na promoção da própria saúde. Diferente de outras áreas de atuação do

psicólogo onde o objetivo é de psicoterapia.

Acerca dos desafios, cabe ressaltar que uma das expectativas descritas no plano de estágio foi a de multidisciplinaridade. Porém, no local foi encontrada certa dificuldade de contato com os profissionais de outros áreas da saúde. Profissionais atuantes na recepção e triagem dos usuários sempre estavam abertos as conversas e trocas. Enquanto alguns acadêmicos, estagiários e professores por vezes dificultavam essa troca. Coube aos estagiários de psicologia demonstrar interesse em construir uma relação profissional entre as áreas.

Quanto as práticas que deverão serem desenvolvidas ao longo do próximo semestre, destaco a importância de seguir o acompanhamento com os pacientes a fim de estabelecer claramente a demanda de cada um deles, e após isso realizar os encaminhamentos necessários para a rede. Ressalto que há possibilidade de intervenção com a equipe multidisciplinar para que ocorram mais reuniões entre todos, com a finalidade de pensar em melhores atendimentos aos usuários. No decorrer do ano, será estudada a criação de grupos com mulheres que possuem fibromialgia, intervenção esta que depende da situação da cidade frente a pandemia do coronavírus. Outras práticas para além dos atendimentos e intervenções com os profissionais deverão ser estudadas, bem como a mapeação de territórios para melhor atender os usuários.

Fora combinado com o professor coordenador local do estágio que, no próximo semestre parte das práticas serão desenvolvida através da organização de materiais e documentos para o ambulatório. Deverá ser construído um meio de comunicação entre os estagiários no Google Drive, onde estes deverão ser acessados apenas com o e-mail institucional, a fim de preservar a identidade dos usuários do serviço. É esperado que neste meio de comunicação sejam criadas pastas com o nome dos estagiários daquele semestre, pacientes que estão em atendimento, locais para encaminhamentos, contatos importantes, fichas de anamnese, entre outros. 


\section{REFERÊNCIAS}

BENEVIDES, R. A Psicologia e o Sistema Único de Saúde: Quais Interfaces? Disponível em: http://www.scielo.br/pdf/psoc/v17n2/27040.

BLEGER, J. Psico-higiene e Psicologia Institucional. Porto Alegre: Artes Médicas, 1992.

BRASIL. Constituição da República Federativa do Brasil. Art.196-200, Brasília, DF, 1988. Brasília, $1988 . \quad$ Disponível em http://conselho.saude.gov.br/web_sus20anos/20anossus/legislacao/constituicaofeder al.pdf.

BRASIL. Lei no 8.080, de 19 de setembro de 1990. Dispõe sobre as condições para a promoção, proteção e recuperação da saúde, a organização e o funcionamento dos serviços correspondentes e dá outras providências. Brasília, 1990. Disponível em http://www.planalto.gov.br/ccivil_03/leis/L8080.htm

BONALDI, C. et al. O trabalho em equipe como dispositivo de integralidade: experiências cotidianas em quatro localidades brasileiras. In: PINHEIRO, Roseni.; Barros, Maria Elisabeth Barros de. Mattos, Ruben Araújo. (Org.). Trabalho em equipe sob o eixo da integralidade: valores, saberes e práticas. 1. ed. Rio de Janeiro: IMS/UERJ: CEPESC: ABRASCO, 2007.

CZERESNIA, Dina. O conceito de Saúde e a Diferença entre Prevenção e Promoção. In: CZERESNIA, Dina. Promoção da saúde: conceitos, reflexões e tendências. Rio de Janeiro, ed. Fiocruz, 2009. 
CONSELHO FEDERAL DE PSICOLOGIA. Práticas e estágios remotos em psicologia no contexto da pandemia da covid-19: recomendações. Recurso Eletrônico. 1 edição. Brasília. CFP, 2020. Disponível em https://site.cfp.org.br/publicacao/praticas-e-estagios-remotos-em-psicologia-no contexto-da-pandemia-da-covid-19-recomendacoes/

CONSELHO FEDERAL DE PSICOLOGIA. Resolução CFP no 010, agosto de 2005. Aprova o Código de Ética Profissional do Psicólogo. 2005. Disponível em https://site.cfp.org.br/wp-content/uploads/2005/07/resolucao2005 10.pdf

RODRIGUES, J. L. da S.; PORTELLA, M.C.; MALIK, A. M. Agenda para a pesquisa sobre o cuidado centrado no paciente no Brasil. Opinião. Revista de Ciência e Saúde $\begin{array}{lllll}\text { Coletiva. } & \text { v.24. } & \text { n.11, } & \text { Disponível em }\end{array}$ https://www.scielo.br/j/csc/a/NbjdSZYLx5yxyLtZ963R7LC/?lang=pt.

OLIVEIRA, G. N. In BRASIL. Ministério da Saúde. Secretaria de Atenção à Saúde. Política Nacional de Humanização. Atenção Básica / Ministério da Saúde, Secretaria de Atenção à Saúde, Política Nacional de Humanização. - 1. ed., 2. reimpr. -Brasília: $\begin{array}{lllll}\text { Ministério da } & \text { Saúde, } & \text { dasponível } & \text { em }\end{array}$ http://bvsms.saude.gov.br/bvs/publicacoes/caderno_humanizasus_atencao_basica_ $\underline{\text { v2 1ed.pdf }}$

SPINK, M. J. P. (Org.) A Psicologia em diálogo com o SUS: prática profissional e produção acadêmica. São Paulo: Casa do Psicólogo, 2010.

YOUNG, J.; KLOSKO, J. S; WEISHAAR, M. E. Terapia do Esquema: Guia de técnicas cognitivo-comportamentais. Porto Alegre: Artmed. 2018. 
MINISTÉRIO DA SAÚDE. Secretaria de Atenção à Saúde. Departamento de Atenção Básica. Acolhimento à demanda espontânea. Brasília, DF: Ministério da Saúde, 2011.

MINISTÉRIO DA SAÚDE. Terminologia básica de saúde. Brasília, Centro de Documentação, 1983. LOPES, A. S. Acolhimento prescrito x real: uma análise sobre as relações entre trabalhadores e usuários na Estratégia Saúde da Família. Dissertação (Mestrado em Saúde da Família) - Centro de Ciências da Saúde, Universidade Federal do Rio Grande do Norte, Natal, 2014.

CZERESNIA, Dina. O conceito de Saúde e a Diferença entre Prevenção e Promoção. In: CZERESNIA, Dina. Promoção da saúde: conceitos, reflexões e tendências. Rio de Janeiro, ed. Fiocruz, 2009. (E-book gratuito via Kindle).

YOUNG, J. E. Cognitive therapyfor perso nality disorders. Sarasota, FL: Professional Re sources Press, 1990.

YOUNG, J. E. The schema diary. New York. Cognitive Therapy Center of New York, 1993.

YOUNG, J. E. Young Parenting Inventory. New York: Cognitive Therapy Center of New York, 1994.

YOUNG, J. E. (1995). Young Compensation Inventory. New York: Cognitive Therapy Center of New York, 1995.

YOUNG, J. E. Cognitive therapy for perso nality disorders: A schema-focused approach (rev. ed.) Sarasota, FL: Professional Resources Press. Artmed Editora sob o título: Tera- pia cognitiva para transtornos da personalidade, 1999.

YOUNG, J. E.; BROWN, G. Young Schema Questionnaire. New York: Cognitive 
Therapy Center of New York, 1990. 